\title{
Coesão familiar associada à saúde bucal, fatores socioeconômicos e comportamentos em saúde
}

\author{
Family cohesion associated with oral health, \\ socioeconomic factors and health behavior
}

Luale Leão Ferreira ${ }^{1}$

Gustavo Antônio Martins Brandão ${ }^{2}$

Gustavo Garcia ${ }^{1}$

Marília Jesus Batista ${ }^{1}$

Ludmila da Silva Tavares Costa ${ }^{1}$

Gláucia Maria Bovi Ambrosano ${ }^{1}$

Rosana de Fátima Possobon ${ }^{1}$

\footnotetext{
${ }^{1}$ Faculdade de Odontologia de Piracicaba, Universidade Estadual de Campinas. Av. Limeira 901, Areião. 13.414-903 Piracicaba SP. lualeleao@yahoo.com.br ${ }^{2}$ Faculdade de Odontologia, Universidade Federal do Pará.
}

\begin{abstract}
Overall health surveys have related family cohesion to socio-economic status and behavioral factors. The scope of this study was to investigate the association between family cohesion and socio-economic, behavioral and oral health factors. This was a, cross-sectional study with twostage cluster sampling. The random sample consisted of 524 adolescents attending public schools in the city of Piracicaba-SP. Variables were evaluated by self-applied questionnaires and caries and periodontal disease were assessed by DMF-T and CPI indices. The adolescent's perception of family cohesion was assessed using the family adaptability and cohesion scale. Univariate and multinomial logistic regression shows that adolescents with low family cohesion were more likely than those with medium family cohesion to have low income (OR 2,28 95\% CI 1,14- 4,55), presence of caries (OR 2,23 95\% CI 1,21-4,09), less than two daily brushings (OR 1,91 95\% CI 1,03-3,54). Adolescents with high family cohesion were more likely than those with medium family cohesion to have high income and protective behavior against the habit of smoking. Thus, the data shows that adolescent perception of family cohesion was associated with behavioral, socio-economic and oral health variables, indicating the importance of an integral approach to patient health.
\end{abstract}

Key words Family relations, Social class, Oral health
Resumo Pesquisas sobre saúde geral têm relacionado coesão familiar a fatores socioeconômicos e comportamentais. O objetivo deste estudo foi investigar a associação entre coesão familiar e fatores socioeconômicos, comportamentais e de saúde bucal. Este foi um estudo transversal com amostra por conglomerados em dois estágios. A amostra randomizada de 524 adolescentes era proveniente de escolas públicas da cidade de Piracicaba-SP. As variáveis foram avaliadas por questionários autoaplicáveis e os dados de saúde bucal, pelos indices CPO e CPI. A coesão familiar percebida pelo adolescente foi avaliada por meio da escala de adaptabilidade e coesão familiar. Análise univariada e regressão logística multinominal mostraram que adolescentes com baixa coesão familiar apresentaram mais chance de terem baixa renda (OR 2,28 IC95\% 1,14-4,55), presença de cárie (OR 2,23 IC95\% 1,21-4,09) e baixa frequência de escovação diária (OR 1,91 IC95\% 1,033,54 ). Adolescentes com alta coesão familiar apresentaram mais chance que adolescentes com média coesão de terem alta renda e fator de proteção contra o hábito de tabagismo. Desta forma, a coesão familiar percebida pelo adolescente associouse com variáveis comportamentais, socioeconômicas e de saúde bucal, indicando a importância de uma abordagem integral da saúde do paciente. Palavras-chave Relações Familiares, Classe social, Saúde bucal 


\section{Introdução}

Em grande parte das sociedades, a família influencia o desenvolvimento infantil e seu impacto continua até a adolescência ${ }^{1,2}$. A influência positiva dos pais sobre seus filhos adolescentes é, muitas vezes, mais efetiva na promoção de comportamentos saudáveis do que a influência dos demais grupos sociais, como amigos ${ }^{2}$.

Devido à importância da família como unidade de atuação em saúde, parte da atenção primária do Sistema Único de Saúde está centrada na Estratégia de Saúde da Família (ESF). A ESF, desde 1994, enfoca a atenção e a assistência à saúde no núcleo familiar, baseando-se, principalmente, nos princípios da promoção da saúde e prevenção de doenças. As unidades de saúde atuam difundindo conhecimento e empoderando as famílias para o enfrentamento de doenças, com o objetivo de promover autonomia e melhorar a qualidade de vida da população atendida ${ }^{3}$.

Neste contexto, a coesão familiar, ou seja, o grau de união entre os familiares, é uma importante unidade de estudo e atuação $0^{4,5}$. A coesão familiar é definida como uma variação entre separação e conexão dos membros da família ou o vínculo emocional entre seus integrantes ${ }^{5}$. Segundo Olson e Gorall ${ }^{5}$, a coesão familiar tem seu foco na relação de equilíbrio entre separação união e dependência - independência entre os sujeitos familiares.

Dependendo do grau de coesão, os familiares exercerão maior ou menor influência entre si, modificando direta e indiretamente a dinâmica e o funcionamento de sua família e influenciando hábitos, comportamentos e crenças ${ }^{5}$. Assim, estudar esta variável pode contribuir para a determinação de grupos vulneráveis a algumas condições de saúde que são influenciadas por fatores comportamentais.

De acordo com os autores da escala que avalia coesão familiar ${ }^{5}$, a alta e a baixa coesão não são consideradas ideais. Os integrantes das famílias com baixa coesão são muito independentes entre si e a estrutura familiar tem pouca influência sobre esses indivíduos. Nas famílias com alta coesão familiar, seus integrantes são extremamente dependentes e apresentam baixa individualidade, o que também não é desejável. Dessa forma, o melhor nível de coesão familiar é o médio, no qual os membros das famílias apresentam uma relação mais harmoniosa e equilibrada entre separação e conexão, dependência e independência.

Segundo a literatura, a coesão familiar está associada a algumas doenças, tais como a asma, o diabetes e a doença crônica renal, que são mediadas por fatores comportamentais ${ }^{6-9}$. A coesão familiar ainda se relaciona com comportamentos inadequados de internalização e de problemas de atenção em crianças $^{10}$, padrão de alimentação e fumo ${ }^{11}$, sintomas de depressão em adolescentes $^{12}$, uso drogas e violência contra a mulher ${ }^{13}$.

Tal como as doenças acima citadas, o surgimento e o agravamento da cárie também podem ser modulados por uma série de fatores. Dentre os já estudados, podem ser destacados os biológicos, como a característica da saliva, a colonização de bactérias patogênicas e os defeitos na estrutura dental ${ }^{14,15}$, os fatores socioeconômicos, como a renda familiar, a escolaridade dos pais e as condições de vida ${ }^{16-20}$ e os fatores comportamentais, como o padrão alimentar, a frequência de consultas anuais ao dentista e de escovação diária ${ }^{21-23}$ e o uso de fluoretos ${ }^{24}$. A doença periodontal, por sua vez, pode ser influenciada por comportamentos como o ineficiente controle do biofilme, a frequência e o consumo de certos alimentos e o hábito de tabagismo ${ }^{25,26}$.

Sabe-se que condições clínicas bucais e orofaciais em crianças têm um forte impacto no âmbito familiar ${ }^{27}$. Porém, a associação de condições clínicas bucais e coesão familiar ainda é um assunto inexplorado pela literatura. Nesta, achados apontam para uma associação entre variáveis socioeconômicas e comportamentais com coesão familiar. A saúde bucal supostamente estaria associada, também, à coesão familiar.

A partir desta hipótese, o objetivo do presente estudo foi investigar os diferentes gradientes da coesão familiar percebida e sua associação com condições socioeconômicas, variáveis comportamentais e clínicas bucais, em adolescentes de 15 anos de escolas públicas da cidade de Piracicaba (SP).

\section{Metodologia}

A amostra foi composta por adolescentes com 15 anos de idade, estudantes de escolas estaduais, que não apresentavam doença sistêmica que pudesse influenciar a doença periodontal (leucemias, Aids, deficiências quantitativas e qualitativas dos neutrófilos). Os pais autorizaram a participação do adolescente na pesquisa por meio da assinatura do termo de consentimento livre e esclarecido.

Primeiramente, obteve-se a permissão para o desenvolvimento da pesquisa junto à Secreta- 
ria Estadual de Educação do município de Piracicaba (SP) e, em seguida, solicitou-se a participação da direção de cada instituição.

O tipo de amostragem utilizada foi probabilística por conglomerado em 2 estágios, escola e aluno. Foram sorteadas 10 dentre as 30 escolas estaduais de ensino médio regularizadas no município. Sua localização foi mapeada a fim de garantir a abrangência das 5 macro-regiões da cidade. Posteriormente, os pesquisadores adquiriram as lista de chamada junto à direção de cada escola, a fim de sortear os alunos que seriam convidados a participar.

A amostra foi calculada baseando-se em um poder do teste (1- $\beta$ ) acima de 0,80 com nível de significância $\alpha$ de 0,05 para um odds ratio de 1,8 , probabilidade de resposta de $12 \%$ e probabilidade de sucesso de $16 \%$, calculada pelo aplicativo SAS Power and Sample Size versão 3.1, o que resultou em uma amostra de, no mínimo, 500 adolescentes. Pensando em possíveis perdas, 725 adolescentes foram convidados a participar da pesquisa.

Os dados foram coletados por meio de exame clínico bucal e questionários autoaplicáveis. Os escolares foram avaliados clinicamente por três examinadores, sob luz natural (sem profilaxia prévia, nem secagem), utilizando a sonda WHO-621 e espelho plano número 5, segundo as recomendações da Organização Mundial da Saúde ${ }^{28}$. Os examinadores foram previamente calibrados por um de referência, com discussões teóricas e práticas, e foi encontrada uma boa reprodutibilidade $^{29}$ interexaminadores com o Kappa $>0,89$ e intraexamiradores com Kappa variando de 0,85 a 1,00 .

As condições clínicas de saúde bucal pesquisadas foram: cárie dentária coronária e condição periodontal, segundo os critérios da $\mathrm{OMS}^{28}$. A experiência de cárie foi avaliada através do índice CPOD, que é obtido pela soma do número de dentes cariados, perdidos e obturados. A condição periodontal foi verificada pelo índice periodontal comunitário (CPI), avaliando a presença de sangramento e cálculo dentário nos dentesíndices 16, 11, 26, 36, 41 e 46.

Cada voluntário examinado respondeu a questionários elaborados para verificação de fatores comportamentais (hábito de tabagismo, tempo em que se deu a última consulta odontológica, frequência de escovação diária, acesso e motivo de consulta odontológica, ansiedade frente ao tratamento odontológico e alimentação em ambiente escolar) adaptados de outros estudos ${ }^{30-}$ ${ }^{32}$. Os questionários foram autoaplicados, assegurando aos participantes a confidencialidade dos dados, para evitar possível viés de informação devido às relações interpessoais dos adolescentes. Em relação aos fatores socioeconômicos, o responsável pelo adolescente respondeu a um questionário validado ${ }^{33}$ abordando renda mensal familiar, grau de escolaridade do pai e da mãe, posse de automóvel, número de residentes na casa e posse de casa própria.

A variável de desfecho deste estudo foi a coesão familiar percebida, avaliada pelo questionário autoplicável FACES III (Family Adaptability and Cohesion Scale), proposto por Olson et al. ${ }^{34}$ e validado no Brasil por Falceto et al..$^{35}$. A escala FACES III propõe investigar o funcionamento e o risco familiar mediante dados de coesão e adaptabilidade familiar. É composta por 20 perguntas, sendo que as questões de número ímpar avaliam a coesão familiar e as questões pares avaliam a adaptabilidade. A cada pergunta é atribuído valor de 1 a 5 , correspondendo o valor 1 a "quase nunca" e o valor 5 a "quase sempre". É realizada a soma dos valores e o escore final pode variar de 10 a 50 para cada domínio.

De acordo com os escores de coesão familiar, Olson et al. ${ }^{34}$ classificaram as famílias em quatro grupos: famílias desligadas, que apresentavam baixo escore de coesão familiar e alta independência entre os familiares; famílias separadas, com escores moderado-baixo de coesão e com certo grau de independência entre os familiares; famílias conectadas, com escores moderado-alto de coesão e moderada dependência entre os familiares; e famílias aglutinadas, que apresentam alto escore de coesão familiar e alto grau de dependência entre os entes familiares.

Segundo Olson et al. ${ }^{34}$, famílias com coesão familiar ideal seriam aquelas que apresentam níveis médios de coesão, ou seja, as famílias separadas e conectadas, onde se tem uma melhor relação entre dependência e independência entre os indivíduos.

No presente estudo, os adolescentes foram divididos de acordo com o desvio padrão da variável coesão familiar percebida em 3 grupos, possibilitando a comparação de grupos de adolescentes com alta e baixa coesão familiar, com o grupo de coesão familiar média. Valores abaixo e acima do desvio-padrão corresponderam às famílias com baixa e alta coesão familiar, respectivamente; e valores entre os desvios-padrão corresponderam a famílias com coesão familiar média.

O desfecho coesão familiar foi avaliado em três categorias: O grupo 1 foi composto pelos adolescentes que apresentaram baixa coesão fa- 
miliar; o grupo 2, adolescentes de famílias com média coesão (grupo referência); e o grupo 3, adolescentes de famílias com alta coesão. Para verificar os fatores associados à coesão familiar (desfecho) foram realizados modelos de Regressão Logística Multinomial, pois a variável dependente possui três categorias.

As variáveis independentes estudadas foram dicotomizadas segundo a mediana ou foram agrupadas em categorias de acordo com a homogeneidade ou com a distribuição das frequências para a verificação da associação entre o desfecho.

A renda familiar, para análise de Regressão Logística Multinominal, foi dividida na mediana, que foi 3 salários míninos. As variáveis posse de casa própria ou de automóvel foram dicotomizadas de acordo com a presença e ausência desses bens. A escolaridade foi classificada, segundo ciclos vigentes, em até oito anos (ensino fundamental) e acima de nove anos (ensino médio incompleto e completo). O número de pessoas residentes na mesma casa foi dividido na mediana, que foi 3. A variável gênero manteve sua classificação original, feminino e masculino.

O hábito de fumar foi verificado neste estudo, por meio do relato do adolescente sobre ter experimentado cigarros ou não. $\mathrm{O}$ acesso ao serviço odontológico foi classificado em nunca ou já passou por consulta odontológica. O tempo da última consulta foi dicotomizado em menos ou mais de seis meses e o motivo da consulta ao dentista foi agrupado por dor ou outros motivos. A frequência de escovação diária foi classificada em até 2 vezes ao dia e mais de duas vezes ao dia.

A informação sobre alimentação do adolescente em ambiente escolar foi dicotomizada em consumo ou não de algum tipo de alimento na escola; alimento cariogênico (doces, biscoitos e guloseimas) ou não.

A informação sobre ansiedade frente ao tratamento odontológico foi dicotomizada em ansiosos e não ansiosos, de acordo a escala avaliativa aplicada ${ }^{30}$.

As condições clínicas de saúde bucal avaliadas foram a presença e a ausência dos componentes $\mathrm{C}, \mathrm{P}$ e O do índice CPOD. O índice CPOD foi dividido de acordo com sua mediana. A condição periodontal foi avaliada de acordo com a presença de sangramento gengival e/ou cálculo em dois ou mais sextantes. Foi também avaliado o terço da população estudada que apresenta a maior experiência de cárie (índice $\mathrm{SiC}$ ), sendo dicotomizada em dois grupos, abrangendo valores menores ou maiores que o valor do Sic in- dex encontrado, segundo recomendações de Nishi et al. ${ }^{36}$. O índice de cuidado odontológico, correspondente ao resultado do componente Obturado $(\mathrm{O})$ dividido pelo total do índice $\mathrm{CPO}$ (O/CPO), foi classificado em diferente de 1 ou igual a $1(\mathrm{O} / \mathrm{CPO} \neq 1$ ou $\mathrm{O} / \mathrm{CPO}=1)$.

Um estudo piloto, utilizando a mesma metodologia desta pesquisa, foi realizado em uma escola não incluída na amostra, com a finalidade verificar a compreensão e as dificuldades dos adolescentes ao responder os questionários utilizados e corrigir os possíveis erros. A amostra foi de 38 adolescentes. O índice CPO-D e o SiC Index encontrados foram 2,18 e 5,54, respectivamente. O percentual de adolescentes livres de cárie foi $36,8 \%(C P O D=0)$. Cerca de 21,05\% (8) dos adolescentes apresentavam dois ou mais sextantes com a presença de cálculo ou sangramento gengival. Um dos instrumentos, que avaliava hábito de tabagismo, foi reformulado para deixar as questões mais objetivas.

Os dados foram tabulados no Statistical Package for the Social Sciences (SPSS) versão $17.0 \mathrm{e}$ no Excell. Primeiramente realizou-se uma análise descritiva obtendo a distribuição absoluta, percentual e desvio padrão (DP) das variáveis.

Foram realizadas análises univariadas das variáveis independentes com a variável dependente, coesão familiar percebida pelo teste quiquadrado e, posteriormente, foram incluídas no modelo de Regressão Logística Multinominal as variáveis que apresentaram $\mathrm{p}<0,25$ pelo método Stepwise Forward.

Este estudo foi realizado de acordo com as Normas e Diretrizes Éticas da Resolução no. 196/ $1996^{37}$ do Conselho Nacional de Saúde do Ministério da Saúde, e aprovado pelo Comitê de Ética em Pesquisa da Faculdade de Odontologia de Piracicaba, Universidade Estadual de Campinas. Todos os responsáveis pelos adolescentes participantes assinaram uma cópia do Termo de Consentimento Livre e Esclarecido para Pesquisa.

\section{Resultados}

Estimando possíveis perdas, foram convidados para participar da pesquisa 725 adolescentes, dos quais 106 se recusaram a participar da pesquisa e 4 foram excluídos pois não satisfaziam os critérios de inclusão do estudo. Foram examinados 615 adolescentes de 15 anos e, devido ao incorreto ou incompleto preenchimento da escala avaliativa de coesão familiar FACES III, 91 adolescentes foram excluídos da análise estatística. A amos- 
tra final, para análise estatística, consistiu em 524 indivíduos de ambos os gêneros.

Do total de 524 participantes, 287 (54,77\%) eram do gênero feminino e 237 (45,22\%) do masculino. O valor médio do índice CPOD e Sic index foi de 1,64 (DP 2,18) e 4,18, respectivamente. Do total da amostra, $47,5 \%(\mathrm{n}=249)$ não tinha experiência de cárie $(\mathrm{CPO}=0)$ e 22,32\% dos adolescentes $(n=117)$ apresentavam lesão cariosa não tratada $(\mathrm{C}>0)$.

A presença de sangramento gengival foi observada em $17,55 \%(\mathrm{n}=92)$ dos adolescentes, sendo que $51(9,73 \%)$ apresentavam dois sextantes ou mais com sangramento ou cálculo dentário.

A média do escore de coesão familiar dos adolescentes foi 32,23, variando do valor 12 a 50. Os adolescentes foram divididos em 3 grupos de acordo com o escore da coesão familiar: grupo com baixa coesão familiar, escore abaixo ou igual a 25,76; grupo com média coesão familiar (grupo referência), escores entre 25,77 e 38,69; e grupo com alta coesão familiar, com escores acima ou igual a 38,70.

A Tabela 1 apresenta a caracterização da população estudada, sua distribuição nos diferentes grupos de coesão familiar e a prevalência das variáveis socioeconômicas, clínicas e comportamentais estudadas.

A Tabela 2 mostra a análise estatística utilizando teste qui-quadrado. As seguintes variáveis mostraram associação estatisticamente significativa com a baixa coesão familiar: renda mensal familiar (<3 salários mínimos), posse de automóvel

Tabela 1. Frequência e porcentagens das variáveis socioeconômicas, comportamentais e clínicas estudadas e prevalência nos diferentes grupos de coesão familiar.

\begin{tabular}{|c|c|c|c|c|}
\hline \multirow[b]{2}{*}{ Variável } & \multirow[b]{2}{*}{$\mathbf{N}$} & \multicolumn{3}{|c|}{ Coesão Familiar } \\
\hline & & $\begin{array}{c}\text { Baixa Coesão } \\
\text { Familiar } \\
\text { n= } 71(\%)\end{array}$ & $\begin{array}{c}\text { Média Coesão } \\
\text { Familiar }^{*} \\
\mathbf{n}=368(\%)\end{array}$ & $\begin{array}{c}\text { Alta Coesão } \\
\text { Familiar } \\
\mathbf{n}=\mathbf{8 5}(\%)\end{array}$ \\
\hline \multicolumn{5}{|l|}{ Gênero } \\
\hline Feminino & 287 & $47(16,37)$ & $197(68,64)$ & $43(14,98)$ \\
\hline Masculino & 237 & $24(10,12)$ & $171(72,15)$ & $42(17,72)$ \\
\hline \multicolumn{5}{|l|}{ Renda familiar } \\
\hline < 3 salários mínimos & 324 & $58(17,90)$ & $223(68,82)$ & $43(13,27)$ \\
\hline > 3 salários mínimos & 200 & $12(6,00)$ & $145(72,5)$ & $42(21,00)$ \\
\hline \multicolumn{5}{|l|}{ Escolaridade do pai } \\
\hline$<8$ anos & 299 & $43(14,38)$ & $221(73,91)$ & $45(15,05)$ \\
\hline$>8$ anos & 225 & $28(12,44)$ & $157(69,77)$ & $40(17,77)$ \\
\hline \multicolumn{5}{|l|}{ Escolaridade da mãe } \\
\hline$<8$ anos & 299 & $44(14,71)$ & $205(68,56)$ & $50(16,72)$ \\
\hline$>8$ anos & 225 & $27(12,00)$ & $163(72,44)$ & $35(15,55)$ \\
\hline \multicolumn{5}{|l|}{ Moradia } \\
\hline Não possui & 180 & $19(10,55)$ & $129(71,66)$ & $32(17,77)$ \\
\hline Possui & 344 & $52(15,11)$ & $239(69,47)$ & $53(15,40)$ \\
\hline \multicolumn{5}{|l|}{ Posse de Automóvel } \\
\hline Não possui & 151 & $33(21,85)$ & $100(66,22)$ & $18(11,92)$ \\
\hline Possui & 373 & $38(10,18)$ & $268(71,84)$ & $67(17,96)$ \\
\hline \multicolumn{5}{|c|}{ Ansiedade Frente ao Tratamento Odontológico (DAS) } \\
\hline Ansiosos & 44 & $10(22,72)$ & $26(13,63)$ & $8(18,18)$ \\
\hline Não ansiosos & 478 & $61(12,76)$ & $341(71,33)$ & $77(16,10)$ \\
\hline \multicolumn{5}{|l|}{ Hábito de tabagismo } \\
\hline Fuma/fumou & 68 & $14(20,58)$ & $51(75.00)$ & $3(4,41)$ \\
\hline Nunca & 456 & $57(12,50)$ & $317(69.51)$ & $82(17,98)$ \\
\hline \multicolumn{5}{|l|}{ Alimentação na escola } \\
\hline Consome alimentos & 451 & $64(14,19)$ & $317(70,28)$ & $73(16,18)$ \\
\hline Não consome nenhum alimento & 70 & $7(10,00)$ & $51(72,85)$ & $12(17,14)$ \\
\hline \multicolumn{5}{|c|}{ Consumo de alimentos cariogênicos em ambiente escolar } \\
\hline Sim & 365 & $43(11,78)$ & $263(72,05)$ & $59(16,16)$ \\
\hline Não & 155 & $27(17,41)$ & $102(65,80)$ & $26(16,77)$ \\
\hline
\end{tabular}




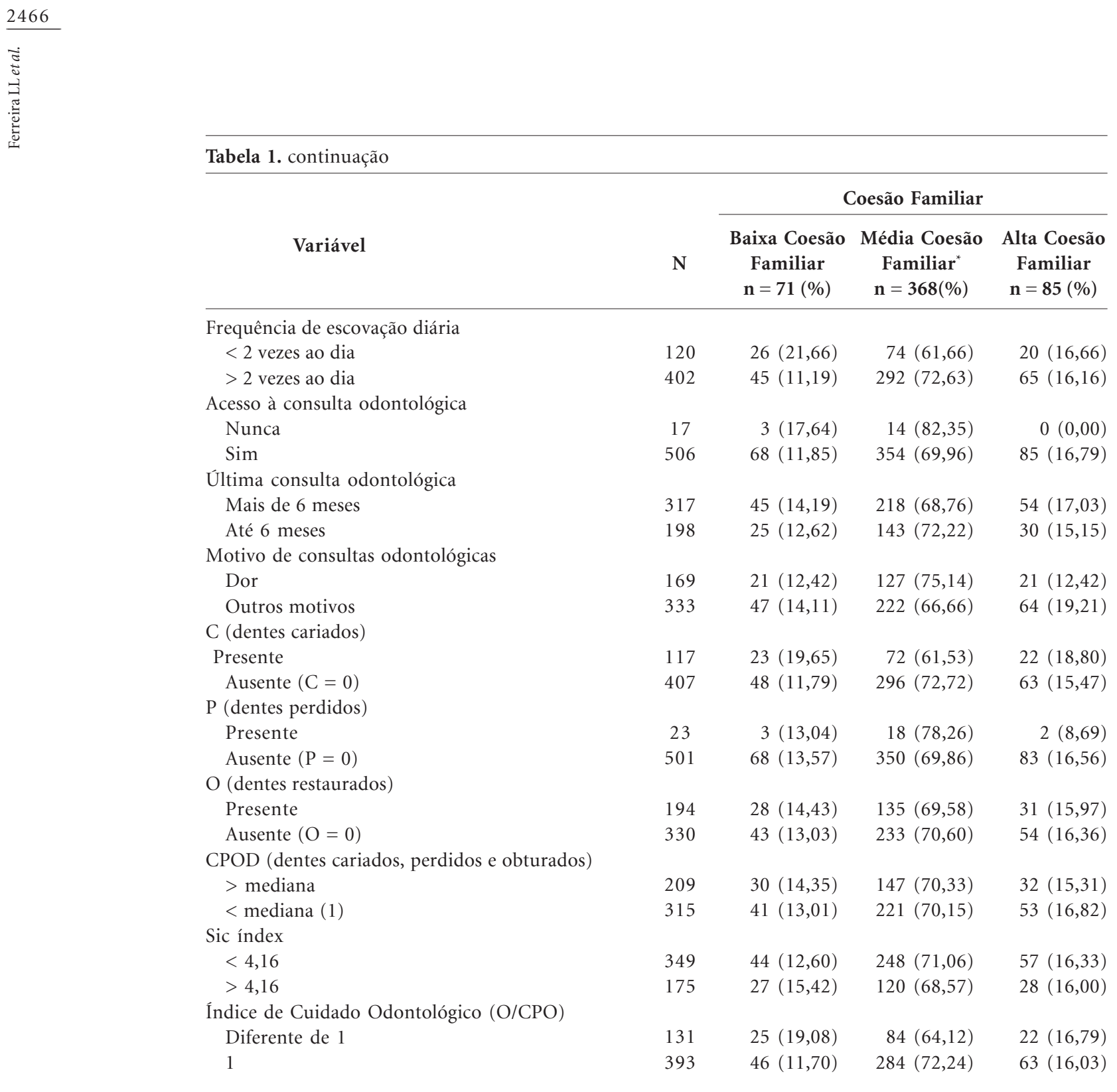

$\mathrm{C}=$ dentes cariados $\mathrm{P}=$ dentes perdidos $\mathrm{O}=$ dentes restaurados $\mathrm{CPOD}=$ soma de dentes cariados, perdidos e obturados; $\mathrm{SiC}$ Index = índice de cárie do terço da amostra com maior prevalência de cárie. ${ }^{\star}$ Grupo de referência.

(não possui), frequência de escovação diária $(<2$ vezes ao dia) e gênero (feminino). Para alta coesão familiar, as variáveis estatisticamente significativas foram renda mensal familiar ( $>3$ salários mínimos) e o hábito de tabagismo (nunca).

Após análise univariada, utilizando teste quiquadrado, todas as variáveis com significância menor que 0,25 foram incluídas no modelo de Regressão Logística Multinominal. A variável posse de carro foi excluída do modelo por ser uma variável colinear à variável renda mensal familiar, sendo esta mantida por apresentar associação mais forte. Após análise estatística e ajuste, e considerando o grupo de média coesão fa- miliar como grupo-referência, as famílias com baixos escores de coesão apresentaram maior chance de baixa renda (OR 2,28 IC95\% 1,14$4,55)$, presença de lesão cariosa não tratada (OR 2,23 IC95\% 1,21-4,09) e baixa frequência de escovação diária (OR 1,91 IC95\% 1,03-3,54). Os adolescentes de famílias com alta coesão familiar apresentaram menor chance de baixa renda mensal (OR 0,59 IC95\% 0,356-0,983) e de hábito de tabagismo (OR 0,21 IC95\% 0,06-0,72) (Tabela 3). As variáveis gênero, consumo de alimentos cariogênicos e última consulta odontológica foram mantidas no modelo devido à sua importância, apesar de não serem significativas. 
Tabela 2. Frequências, porcentagens e intervalo de confiança ao nível de 95\%, Odds Ratio bruto e análise da associação dos grupos de coesão familiar com as variáveis socioeconômicas, comportamentais e clínicas bucais pelo teste Qui-quadrado.

\begin{tabular}{|c|c|c|c|c|}
\hline \multirow{2}{*}{ Variáveis } & \multirow[b]{2}{*}{$\mathbf{n}$} & \multicolumn{3}{|c|}{ Baixa Coesão Familiar } \\
\hline & & n (\%) & OR Bruto (IC95\%) & $\mathbf{p}$ \\
\hline \multicolumn{5}{|l|}{ Renda } \\
\hline$<3$ salários & 324 & $58(17,9 \%)$ & $2,9(1,54-5,48)$ & 0,00 \\
\hline > 3 salários & 200 & $12(6 \%)$ & 1 & \\
\hline \multicolumn{5}{|l|}{ Carro } \\
\hline Não possui & 151 & $33(21,85 \%)$ & $2,38(1,38-3,91)$ & 0,00 \\
\hline Possui & 373 & $38(10,18 \%)$ & 1 & \\
\hline \multicolumn{5}{|l|}{ Hábito de tabagismo } \\
\hline Fuma/fumou & 68 & $14(20,58 \%)$ & $1,52(0,79-2,93)$ & 0,20 \\
\hline Nunca & 456 & $57(12,5 \%)$ & 1 & \\
\hline \multicolumn{5}{|l|}{ Cárie } \\
\hline Presente & 117 & $23(19,65 \%)$ & $1,97(1,15-3,44)$ & 0,01 \\
\hline Ausente $(c=0)$ & 407 & $48(11,79 \%)$ & 1 & \\
\hline \multicolumn{5}{|l|}{ Frequência de escovação diária } \\
\hline$<2$ vezes ao dia & 120 & $26(21,66 \%)$ & $2,28(1,32-3,94)$ & 0,00 \\
\hline$>2$ vezes ao dia & 402 & $45(11,19 \%)$ & 1 & \\
\hline \multicolumn{5}{|c|}{ Índice de Cuidado Odontológico (O/CPO) } \\
\hline$<1$ & 131 & $25(19,08 \%)$ & $1,83(1,06-3,16)$ & 0,02 \\
\hline 1 & 393 & $46(11,70 \%)$ & 1 & \\
\hline \multicolumn{5}{|l|}{ Gênero } \\
\hline Feminino & 287 & $47(16,37 \%)$ & $1,75(1,02-3,0)$ & 0,04 \\
\hline Masculino & 237 & $24(10,12 \%)$ & 1 & \\
\hline \multicolumn{5}{|c|}{ Ansiedade Frente ao Tratamento Odontológico (DAS) } \\
\hline Ansiosos & 44 & $10(22,72 \%)$ & $2,15(0,98-4,68)$ & 0,05 \\
\hline Não ansiosos & 478 & $61(12,76 \%)$ & 1 & \\
\hline \multicolumn{5}{|c|}{ Consumo de alimentos cariogênicos em ambiente escolar } \\
\hline Sim & 365 & $43(11,78 \%)$ & $1,61(0,95-2,75)$ & 0,07 \\
\hline Não & 159 & $28(17,61 \%)$ & 1 & \\
\hline \multicolumn{5}{|l|}{ Última consulta odontológica } \\
\hline Mais de 6 meses & 317 & $45(14,19 \%)$ & $1,53(0,88-2,64)$ & 0,12 \\
\hline Até 6 meses & 198 & $25(12,62 \%)$ & 1 & \\
\hline \multicolumn{5}{|l|}{ Motivos de consultas odontológicas } \\
\hline Dor & 169 & $21(12,42 \%)$ & $0,77(0,39-1,50)$ & 0,44 \\
\hline Outros motivos & 333 & $47(14,11 \%)$ & 1 & \\
\hline
\end{tabular}

\section{Discussão}

O presente estudo investiga a associação de condições clínicas bucais, variáveis de comportamentos em saúde e socioeconômicas com coesão familiar, assunto ainda pouco explorado pela literatura. Andrade et al. ${ }^{38}$, em pesquisa abordando idosos brasileiros, estudou a ingestão inadequada de nutrientes e sua associação com condição de saúde bucal e coesão familiar. Este estudo, apesar de abordar saúde bucal e coesão familiar, não investigou a associação direta entre coesão familiar e saúde bucal. 
Tabela 2. continuação

\begin{tabular}{|c|c|c|}
\hline \multirow{2}{*}{ Variáveis } & \multirow[b]{2}{*}{$\mathbf{n}$} & \multirow{2}{*}{$\begin{array}{c}\text { Média Coesão Familiar } \\
\mathbf{n}(\%)\end{array}$} \\
\hline & & \\
\hline \multicolumn{3}{|l|}{ Renda } \\
\hline$<3$ salários & 324 & $223(68,83 \%)$ \\
\hline$>3$ salários & 200 & $145(72,5 \%)$ \\
\hline \multicolumn{3}{|l|}{ Carro } \\
\hline Não possui & 151 & $100(66,22 \%)$ \\
\hline Possui & 373 & $268(71,84 \%)$ \\
\hline \multicolumn{3}{|l|}{ Hábito de tabagismo } \\
\hline Fuma/fumou & 68 & $51(75 \%)$ \\
\hline Nunca & 456 & $317(69,51 \%)$ \\
\hline \multicolumn{3}{|l|}{ Cárie } \\
\hline Presente & 117 & $72(61,53 \%)$ \\
\hline Ausente $(c=0)$ & 407 & $296(72,72 \%)$ \\
\hline \multicolumn{3}{|l|}{ Frequência de escovação diária } \\
\hline$<2$ vezes ao dia & 120 & $74(61,66 \%)$ \\
\hline$>2$ vezes ao dia & 402 & $292(72,63 \%)$ \\
\hline \multicolumn{3}{|c|}{ Índice de Cuidado Odontológico (O/CPO) } \\
\hline$<1$ & 131 & $84(64,12 \%)$ \\
\hline 1 & 393 & $284(72,26 \%)$ \\
\hline Gênero & & 1 \\
\hline Feminino & 287 & $97(68,64 \%)$ \\
\hline Masculino & 237 & $171(72,15 \%)$ \\
\hline \multicolumn{3}{|c|}{ Ansiedade Frente ao Tratamento Odontológico (DAS) } \\
\hline Ansiosos & 44 & $26(59,09 \%)$ \\
\hline Não ansiosos & 478 & $341(71,33 \%)$ \\
\hline \multicolumn{3}{|c|}{ Consumo de alimentos cariogênicos em ambiente escolar } \\
\hline Sim & 365 & $263(72,05 \%)$ \\
\hline Não & 159 & $102(64,15 \%)$ \\
\hline \multicolumn{3}{|l|}{ Última consulta odontológica } \\
\hline Mais de 6 meses & 317 & $218(68,76 \%)$ \\
\hline Até 6 meses & 198 & $143(72,22 \%)$ \\
\hline \multicolumn{3}{|l|}{ Motivos de consultas odontológicas } \\
\hline Dor & 169 & $127(75,14 \%)$ \\
\hline Outros motivos & 333 & $222(66,66 \%)$ \\
\hline
\end{tabular}

continua

coesão familiar, comparado ao grupo que apresenta coesão familiar média e equilibrada.

A renda mensal familiar mostrou-se significativa tanto no grupo de baixa quanto no grupo de alta coesão familiar, podendo ser considerada, em adolescentes de baixa renda, como indicador de risco para baixa coesão familiar. Os adolescentes de alta renda tiveram maior chance de pertencer ao grupo de alta coesão familiar. No entanto, esta relação é pouco abordada, uma vez que os estudos que avaliam coesão familiar geralmente o fazem em grupos de risco, sendo utilizada nos modelos estatísticos como variável independente. Os estudos que investigam renda mensal ou anual familiar geralmente descrevem a distribuição de renda para caracterização da amostra e não a relacionam com a coesão familiar ${ }^{40}$.

Porém, Mandara e Murray ${ }^{41}$ relataram a relação estatística significativa entre renda e funcionamento familiar. Outros estudos sugerem que algumas inadequações de comportamento manifestados por famílias monoparentais não são devidas à ausência física da figura de um dos pais, mas sim à falta de recursos econômicos gerado pelo responsável ausente ${ }^{42}$.

Apesar de não ter nenhuma ligação direta com a presença clínica de doença bucal, os adolescentes com lesão cariosa não tratada apresentam maior chance de ter baixa coesão familiar após ajuste das variáveis. A baixa coesão familiar ain- 
Tabela 2. continuação

\begin{tabular}{|c|c|c|c|c|}
\hline \multirow{2}{*}{ Variáveis } & \multirow[b]{2}{*}{$\mathbf{n}$} & \multicolumn{3}{|c|}{ Alta Coesão Familiar } \\
\hline & & n (\%) & OR Bruto (IC95\%) & $\mathbf{p}$ \\
\hline \multicolumn{5}{|l|}{ Renda } \\
\hline$<3$ salários & 324 & $43(13,27 \%)$ & $0,66(0,41-1,07)$ & 0,09 \\
\hline > 3 salários & 200 & $42(21,0 \%)$ & 1 & \\
\hline \multicolumn{5}{|l|}{ Carro } \\
\hline Não possui & 151 & $18(11,92 \%)$ & $0,72(0,40-1,27)$ & 0,26 \\
\hline Possui & 373 & $67(17,96 \%)$ & 1 & \\
\hline \multicolumn{5}{|l|}{ Hábito de tabagismo } \\
\hline Fuma/fumou & 68 & $3(4,41 \%)$ & $0,22(0,06-0,74)$ & 0,01 \\
\hline Nunca & 456 & $82(17,98 \%)$ & 1 & \\
\hline \multicolumn{5}{|l|}{ Cárie } \\
\hline Presente & 117 & $22(18,80 \%)$ & $1,43(0,82-2,48)$ & 0,19 \\
\hline Ausente $(c=0)$ & 407 & $63(15,47 \%)$ & 1 & \\
\hline \multicolumn{5}{|l|}{ Frequência de escovação diária } \\
\hline$<2$ vezes ao dia & 120 & $20(16,66 \%)$ & $1,21(0,69-2,13)$ & 0,49 \\
\hline$>2$ vezes ao dia & 402 & $65(16,16 \%)$ & 1 & \\
\hline \multicolumn{5}{|c|}{ Índice de Cuidado Odontológico (O/CPO) } \\
\hline$<1$ & 131 & $22(16,79 \%)$ & $1,18(0,68-2,03)$ & 0,54 \\
\hline 1 & 393 & $63(16,03 \%)$ & 1 & \\
\hline \multicolumn{5}{|l|}{ Gênero } \\
\hline Feminino & 287 & $43(14,98 \%)$ & $0,90(0,56-1,41)$ & 0,44 \\
\hline Masculino & 237 & $42(17,72 \%)$ & 1 & \\
\hline \multicolumn{5}{|c|}{ Ansiedade Frente ao Tratamento Odontológico (DAS) } \\
\hline Ansiosos & 44 & $8(18,18 \%)$ & $1,38(0,60-3,26)$ & 0,44 \\
\hline Não ansiosos & 478 & $77(16,10 \%)$ & 1 & \\
\hline \multicolumn{5}{|c|}{ Consumo de alimentos cariogênicos em ambiente escolar } \\
\hline Sim & 365 & $59(16,16 \%)$ & $1,13(0,67-1,90)$ & 0,62 \\
\hline Não & 159 & $26(16,35 \%)$ & 1 & \\
\hline \multicolumn{5}{|l|}{ Última consulta odontológica } \\
\hline Mais de 6 meses & 317 & $54(17,03 \%)$ & $0,88(0,52-1,49)$ & 0,64 \\
\hline Até 6 meses & 198 & $30(15,15 \%)$ & 1 & \\
\hline \multicolumn{5}{|l|}{ Motivos de consultas odontológicas } \\
\hline Dor & 169 & $21(12,42 \%)$ & $0,54(0,27-1,07)$ & 0,07 \\
\hline Outros motivos & 333 & $64(19,21 \%)$ & 1 & \\
\hline
\end{tabular}

"Grupo de referência.

da foi associada com a baixa frequência na escovação diária, podendo supor que em famílias menos coesas há uma menor propagação de comportamentos em saúde, como seria o caso do hábito de higiene dental. A presença de biofilme dental, devido à baixa frequência de higienização, é um dos fatores etiológicos da cárie e pode estar associada com a maior prevalência desta doença no grupo de menor coesão. Os adolescentes de famílias com baixos escores de coesão apresentam higienização bucal escassa, comportamento que contribui para o surgimento de cá$\mathrm{rie}^{21,23}$. Além da higienização bucal escassa, a baixa coesão familiar também foi associada à baixa renda mensal, considerado outro fator de risco para desenvolvimento de cárie ${ }^{18,33}$. A relação de coesão familiar e cárie dentária se dá, portanto, por via indireta.

O grupo com alta coesão familiar apresentou menor chance de baixa renda mensal (OR 0,59 IC95\% 0,35-0,98) e de hábito de tabagismo (OR 0,21 IC95\% 0,06-0,72). Em relação ao uso de cigarros, os achados corroboram o estudo de Franko et al. ${ }^{11}$, que discute que as famílias que apresentam alta coesão familiar oferecem maior suporte ao adolescente, propiciando melhores escolhas, voltadas para um comportamento saudável contra o uso de tabaco. 
Tabela 3. Associação entre variáveis socioeconômicas, comportamentais e clínicas bucais nos grupos polarizados de coesão familiar através de Análise de Regressão Logística Multinominal em relação ao grupo de Média Coesão Familiar (grupo referência).

\begin{tabular}{|c|c|c|c|c|}
\hline & \multicolumn{4}{|c|}{ Baixa Coesão Familiar } \\
\hline & OR Bruto (IC95\%) & $\mathbf{P}$ & OR ajustado (IC 95\%) & $\mathbf{P}$ \\
\hline \multicolumn{5}{|l|}{ Renda } \\
\hline$<3$ salários & $2,90(1,54-5,48)$ & 0,00 & $2,28(1,14-4,55)$ & $0,01^{*}$ \\
\hline$>3$ salários & 1,0 & & & \\
\hline \multicolumn{5}{|l|}{ Hábito de tabagismo } \\
\hline Fuma/fumou & $1,52(0,79-2,93)$ & 0,20 & $1,26(0,57-2,77)$ & 0,56 \\
\hline Nunca & 1,0 & & & \\
\hline \multicolumn{5}{|l|}{ Cárie } \\
\hline Presente & $1,97(1,15-3,44)$ & 0,01 & $2,23(01,21-4,09)$ & $0,00^{\star}$ \\
\hline Ausente $(c=0)$ & 1,0 & & & \\
\hline \multicolumn{5}{|c|}{ Frequência de escovação diária } \\
\hline$<2$ vezes ao dia & $2,28(1,32-3,93)$ & 0,003 & $1,91(1,03-3,54)$ & $0,03^{*}$ \\
\hline$>2$ vezes ao dia & 1,0 & & & \\
\hline \multicolumn{5}{|l|}{ Gênero } \\
\hline Feminino & $1,75(1,02-3,00)$ & 0,041 & $1,63(0,91-2,93)$ & 0,10 \\
\hline Masculino & 1,0 & & & \\
\hline \multicolumn{5}{|c|}{ Consumo de alimentos cariogênicos } \\
\hline Sim & $1,61(0,95-2,75)$ & 0,07 & $1,7(0,94-3,06)$ & 0,07 \\
\hline Não & 1,0 & & & \\
\hline \multicolumn{5}{|c|}{ Última consulta odontológica } \\
\hline Mais de 6 meses & $1,53(0,88-2,64)$ & 0,125 & $1,25(0,69-2,24)$ & 0,45 \\
\hline \multirow[t]{3}{*}{ Até 6 meses } & 1,0 & & & \\
\hline & & \multicolumn{3}{|c|}{ Alta Coesão Familiar } \\
\hline & OR Bruto (IC95\%) & $\mathbf{p}$ & OR ajustado (IC 95\%) & $\mathbf{P}$ \\
\hline \multicolumn{5}{|l|}{ Renda } \\
\hline$<3$ salários & $0,66(0,41-1,06)$ & 0,09 & $0,59(0,35-0,98)$ & $0,04^{*}$ \\
\hline > 3 salários & 1,0 & & & \\
\hline \multicolumn{5}{|l|}{ Hábito de tabagismo } \\
\hline Fuma/fumou & $0,22(0,06-0,74)$ & 0,01 & $0,21(0,06-0,72)$ & $0,01^{*}$ \\
\hline Nunca & 1,0 & & & \\
\hline \multicolumn{5}{|l|}{ Cárie } \\
\hline Presente & $1,43(0,82-2,48)$ & 0,19 & $1,71(0,96-3,04)$ & 0,06 \\
\hline Ausente $(c=0)$ & 1,0 & & & \\
\hline Frequência de esc & & & & \\
\hline$<2$ vezes ao dia & $1,21(0,69-2,13)$ & 0,49 & $1,32(0,73-2,39)$ & 0,35 \\
\hline$>2$ vezes ao dia & 1,0 & & & \\
\hline \multicolumn{5}{|l|}{ Gênero } \\
\hline Feminino & $0,90(0,56-1,41)$ & 0,44 & $1,07(0,65-1,77)$ & 0,77 \\
\hline Masculino & 1,0 & & & \\
\hline \multicolumn{5}{|c|}{ Consumo de alimentos cariogênicos } \\
\hline Sim & $1,13(0,67-1,90)$ & 0,62 & $1,01(0,58-1,76)$ & 0,94 \\
\hline Não & 1,0 & & & \\
\hline \multicolumn{5}{|c|}{ Última consulta odontológica } \\
\hline Mais de 6 meses & $0,88(0,52-1,49)$ & 0,64 & $0,88(0,51-1,54)$ & 0,67 \\
\hline Até 6 meses & 1,0 & & & \\
\hline
\end{tabular}

${ }^{*} \mathrm{p}<0,05$ 
Pode-se observar, também, que o universo estudado corresponde a adolescentes de escolas públicas, havendo menor diferença nos fatores externos que poderiam levar a viés em estudos de coesão familiar. Entretanto, a abordagem somente de adolescentes de escolas públicas é uma limitação do estudo, fazendo-se necessário avaliar e entender como a coesão familiar se comportaria entre adolescentes de escolas particulares. Além disso, pode-se sugerir uma abordagem longitudinal do estudo, analisando a coesão familiar e as variáveis comportamentais e de saúde bucal, visando à adequada avaliação do comportamento de outros fatores ao longo do curso de vida, buscando investigar e estabelecer a possível relação entre as mesmas.

Por se tratar de uma pesquisa de natureza transversal, este estudo não visa estabelecer relação causal entre as variáveis e coesão familiar. Ao considerar a coesão familiar como variável de desfecho, pretendia-se investigar como, em diferentes gradientes, está associada a aspectos sociais, econômicos, comportamentais e de saúde, verificando se o contexto familiar é um aspecto importante a ser considerado em estudos dos processos de saúde/doença.

No presente estudo, a coesão familiar apresenta uma relação gradiente, ou seja, no grupo de menor coesão familiar são observadas piores condições clínicas e comportamentais. A situação contrária ainda se faz verdadeira, na qual o grupo com maior coesão apresenta condições clínicas favoráveis e maior número de comportamentos em saúde. Esse resultado contradiz os autores da escala diagnóstica FACES III, que propõe o modelo circumplexo, no qual as famílias com escores médios de coesão familiar seriam as mais equilibradas e apresentariam uma relação mais harmoniosa entre dependência e independência e seriam, portanto, mais saudáveis.

Outros estudos, porém, têm usado a escala diagnóstica, criticando o modelo circumplexo proposto por Olson et al. ${ }^{34}$ e reafirmando a relação gradiente entre a coesão familiar e um melhor funcionamento familiar, ratificando os resultados encontrados no presente estudo ${ }^{43,44}$. Farrel e Barnes $^{43}$ afirmaram que a relação gradiente da escala avaliativa FACES III pode ser encontrada em estudos abordando aspectos psicológicos, comportamentais e de qualidade de relacionamentos, indicando a uso da escala avaliativa para pesquisas de suporte social no ambiente familiar.

Em estudos abordando coesão familiar e desfechos de saúde, Hanson et al. ${ }^{45}$ avaliaram a relação entre coesão familiar e tempo de diagnóstico de diabetes em adolescentes insulino-dependentes. Os autores observaram que nos pacientes com pouco tempo de diagnóstico de diabetes, as famílias com baixa coesão familiar foram relacionadas com pobre controle metabólico, enquanto nas famílias de pacientes com maior tempo de diagnóstico, a coesão familiar não variou de acordo com o padrão de controle metabólico, relatando a complexidade da relação.

Num estudo com pacientes com asma crôni$\mathrm{ca}^{6}$ foi observado que crianças que apresentaram coesão familiar média tinham valores mais altos de imunoglobulina IgE quando comparadas com outras de grupos extremos de coesão familiar. Porém, este estudo agrupou crianças de baixa e alta coesão familiar, não considerando as características funcionais opostas entre as famílias de baixa e alta coesão e também não comparou estes grupos entre si.

Os resultados do presente estudo sugerem a incorporação da variável de coesão familiar nas pesquisas de saúde bucal, devido à sua associação com renda mensal familiar e frequência de higiene bucal - conhecidos fatores de risco à cárie dentaria - e com a presença de lesão de cárie. Ao incorporar a avaliação do ambiente e da força das relações familiares nos estudos de saúde bucal, contempla-se um aspecto que pode ser de grande importância em levantamentos epidemiológicos, buscando integrar saúde bucal nos demais programas sociais e de saúde, empoderando comunidades ${ }^{46}$. A coesão familiar é uma variável pouco explorada na literatura e não existem estudos que investigam a associação entre saúde bucal e coesão familiar.

\section{Conclusão}

Concluiu-se que a coesão familiar percebida pelo adolescente foi associada a variáveis comportamentais, a saúde bucal e a fatores socioeconômicos, indicando a importância de avaliação das relações familiares em estudos abordando saúde bucal.

A coesão familiar apresentou uma relação gradiente com os fatores associados, no qual os adolescentes com baixa coesão familiar apresentaram piores condições socioeconômicas, maior quantidade de comportamentos prejudiciais à saúde e maior número de lesões cariosas não tratadas; e adolescentes com alta coesão familiar mostraram ter melhores condições socioeconômicas e menor exposição (fator de proteção) ao hábito de tabagismo. 


\section{Colaboradores}

LL Ferreira participou da concepção, da pesquisa, da metodologia e da redação final; GAM Brandão participou da concepção, da pesquisa e da redação final do artigo; G Garcia colaborou na pesquisa e na metodologia; MJ Batista participou da metodologia da análise e interpretação dos dados; LST Costa participou da redação final do artigo; GMB Ambrosano colaborou na metodologia e na análise estatística; e RF Possobon participou da concepção, da metodologia e na redação final.

\section{Agradecimentos}

Os autores agradecem à Diretoria de Ensino da cidade de Piracicaba-SP, que autorizou a realização da pesquisa, às escolas e aos alunos participantes. À Faculdade de Odontologia de Piracicaba-Unicamp, em especial aos professores Fábio Luiz Mialhe e Antonio Carlos Pereira pelas colaborações. Agradecemos também a Janice Simpson de Paula e a Luísa Helena do Nascimento Tôrres pelas observações e auxilio na correção do artigo.

\section{Referências}

1. Resnick MD, Bearman OS, Blum RW, Bauman KE, Harris KM, Jones J, Tabor J, Beuhing T, Sieving RE, Shew M, Ireland M, Bearinger LH, Udry R. Protecting Adolescents from harm. Findings from the National Longitudinal Study on Adolescent Health. J Am Med Ass 1997; 278(10):823-832.

2. Joronen K, Åstedt-Kurki P. Familial contribution to adolescent subjective well-being. Int J Nurs Pract 2005; 11(3):125-133.

3. Brasil. Ministério da Saúde (MS). Secretaria executiva. Programa Saúde da Família - PSF. Brasília: MS; 2001.

4. Roosa MW, Dunka L, Tein JY. Family characteristics as mediators of the influence of problem drinking and multiple risk status on child mental health. Am J Community Psychology 1996; 24(5):607-624.

5. Olson DH, Gorall DM. Circumplex Model of marital and family systems. In: Walsh F, editors. Normal Family Processes. $3^{\text {th }}$ Edition. New York: Guilford; 2003. p. 514-547.

6. Gustafsson PA, Kjellman NIM, Ludvigsson J, Cederblad M. Asthma and family interaction. Arch Dis Child 1987; 62(3):258-263.

7. Konen JC, Sumerson JH, Dignan MB. Family function, stress and locus of control. Arch Fam Med 1993; 2(4):393-402.

8. Holden EW, Chmielewski D, Nelson CC, Kager VA. Contolling for general and disease-specific effects in child and family adjustment to chronic childhood illness. J Pediat Psychol 1997; 22(1):15-27.

9. Soliday E, Kool E, Lande MB. Family environment, child baharior, and medical indicators in children with kidney disease. Child Psychiatry Hum Dev 2001; 31(4):279-295

10. Lucia VC, Breslau N. Family Cohesion and children's behavior problems: a longitudinal investigation. Psychiatry Res 2005; 141(2):141-149.

11. Franko DL, Thompson D, Affenito SG, Barton BA. What mediates the relationship between family meals and adolescent health issues? Health Psychol 2008; 27(2):109-117.

12. Garrison CZ, Waller JL, Cuffe SP McKeown RE, Addy $\mathrm{CL}$, Jackson KL. Incidence of major depressive disorder and dysthymia in young adolescents. $J A m$ Acad Child Adolesc Psychiatry 1997; 36(4):458-465.

13. Rabelo PM, Caldas-Júnior AF. Violência contra a mulher, coesão familiar e drogas. Rev Saude Publica 2007; 41(6):970-978.

14. Loeshe WJ. Role of Streptococcus mutans in Human Dental Decay. Microbiol Rev 1986; 50(4):353-380.

15. Featherstone JDB. The science and practice of caries prevention. J Am Dent Assoc 2000; 131(7):887-899.

16. Antunes JLF, Narvai PC, Nugent NZ. Measuring inequalities in the distribution of dental caries. Community Dent Oral Epidemiol 2004; 32:(1)41-48.

17. Locker D, Frosina C, Murray H, Wiebe D, Wiebe P. Identifying children with dental care needs: evaluation of a targeted school-based dental screening program. J Pub Health Dent 2004; 64(2):63-70.

18. Pereira SM, Tagliaferro EPS, Ambrosano GMB, Cortellazzi KL, Meneghim MC, Pereira AC. Dental caries in 12-year-old Schoolchildren and its relationship with socioeconomic and behavioural variables. Oral Health Prev Dent 2007; 5(4):299-306. 
19. Petersen PE. Sociobehavioural risk factors in dental caries - international perspectives. Community Dent Oral Epidemiol 2005; 33(4):274-279.

20. Pattussi MP, Marcenes W, Croucher R, Sheiham A. Social deprivation, income inequality, social cohesion and dental caries in Brazilian school children. Social Sci Med 2001; 53(7):915-925.

21. Mascarenhas AK. Oral hygiene as a risk indicator of enamel and dentin caries. Community Dent Oral Epidemiol 1998; 26(5):331-339.

22. Gibson S, Williams S. Dental Caries in Pre-School Children: Associations with Social Class, Toothbrushing Habit and Consumption of Sugars and Sugar-Containing Foods. Caries Res 1999; 33(2):101113.

23. Levin L, Shenkman A. The Relationship Between Dental Caries Status and Oral Health Attitudes and Behavior in Young Israeli Adults. J Dent Educ 2004; 68(11):1185-1191.

24. Cury JA, Tenuta LMA, Ribeiro CCC, Paes Leme AF. The importance of fluoride dentifrices to the dental caries prevalence in Brazil. Braz Dent J 2004; 15(3):167-174

25. Bergstrom J, Eliasson S, Dock J. A 10-year prospective study of tobacco smoking and periodontal health. J Periodontal 2000; 71(8):1338-1347.

26. Löe H, Theilade E, Jensen SE. Experimental gingivitis in man. J Periodontal 1965; 36(5):177-187.

27. Locker D, Jokovik A, Stephens M, Kenny D, Thompson B, Guyatt G. Family impact of child oral and oro-facial condictions. Community Dent Oral Epidemiol 2002; 30(6):438-448.

28. World Health Organization (WHO), FDI World Dental Federation. Oral health surveys. Basic methods. Geneva: Print Reports; 1997.

29. Peres MA, Traebert J, Marcenes W. Calibração de examinadores para estudos epidemiológicos de cárie dentária. Cad Saude Publica 2001; 17(1):153-159.

30. Corah NL, Gale EN, Illig SJ. Assessment of a dental anxiety scale. J Am Dent Assoc 1978; 97(5):816-819.

31. Farias-Júnior JC, Pires, MC, Lopes AS. Reprodutibilidade de um questionário para o levantamento de informações sobre comportamentos relacionados à saúde em adolescentes. Rev Bras Cien Mov 2002; 10(3):43-48.

32. Lisboa IC, Abegg C. Hábitos de higiene bucal e uso de serviços odontológicos por adolescentes e adultos do Município de Canoas, Estado do Rio Grande do Sul, Brasil. Epidem Serv Saude 2006; 15(4):29-39.

33. Meneghim MC, Kozlowski FC, Pereira AC, Ambrosano GMB, Meneghim ZMAP. Classificação socioeconômica e sua discussão em relação à prevalência de cárie e fluorose dentária. Cien Saude Colet 2007; 12(2):523-529.

34. Olson DH, Sprenkle D, Russel C. Cicumplex model: systemic assessment and treatment of families. New York: The Harworth press; 1989.

35. Falceto OG, Busnell ED, Bozzetti MC. Validação de escalas diagnósticas do funcionamento familiar para a utilização em serviços de atenção primária à saúde. Pan Am J Public Health 2000; 7(4):255-263.
36. Nishi M, Bratthall D, Stjernswärd J. How to calculate the Significant Caries Index (SiC Index). Malmö: WHO Collaborating Centre, University of Malmö; 2001.

37. Brasil. Ministério da Saúde(MS). Conselho Nacional de Saúde. Resolução no. 196 de 10 de outubro de 1996. Diretrizes e Normas Regulamentadoras de Pesquisas Envolvendo Seres Humanos. Diário Oficial da União 1996; 16 out.

38. Andrade FB, Caldas-Júnior AF, Kitoko PM, Zandonade E. The relationship between nutriente intake, dental status and Family cohesion among older brazilians. Cad Saude Publica 2011; 27(1):113-122.

39. Cangussu MCT, Castelhanos RA, Pinheiro MF, Albuquerque SR, Pinho C. Cárie dentária em escolares de 12 e 15 anos de escolas públicas e privadas de Salvador, Bahia, Brasil, em 2001. Pesqui Odontol Bras 2002; 16(4):379-384.

40. Miranda AO, Estrada D, Jimenez MF. Differences in Family Cohesion, Adaptability, and Environment among Latino Families in dissimilar Stages of acculturation. The Family Journal 2000; 8(4):341-350.

41. Mandara J, Murray CB. Effects of Parental Marital Status, Income, and Family Functioning on African American Adolescent Self-Esteem. J Fam Psychol 2000; 14(3):475-490.

42. McLeod J, Kruttschnitt C, Dornfeld M. Does parenting explain the effects of structural conditions on children's antisocial behavior? A comparison of Blacks and Whites. Social Forces 1994; 73(2):575-604.

43. Farrell MP, Barnes GM. Family Systems and Social Support: a test of the effects of cohesion and adaptability on the functioning of parents and adolescents. J Marriage and family 1993; 55(1):119-132.

44. Barber BK, Buehler C. Family cohesion and enmeshment: different constructs, different effects. J Marriage and family 1996; 58(5):433-441.

45. Hanson CL, Henggeler SW, Harris MA. Family system variables and the health status of adolescents with insulin-dependent diabetes mellitus. Health Psychol 1989; 8(2):239-253.

46. 46. Mouradian WE, Huebner CE, Ramos-Gomez F, Slavkin HC. Beyond Access: The Role of Family and Community in Children's Oral Health. J Dent Education 2007; 71(5):619-631.

Artigo apresentado em 18/08/2012

Aprovado em 10/10/2012

Versão final apresentada em 29/10/2012 
ERRATA

Errata do artigo Coesão familiar associada à saúde bucal, fatores socioeconômicos

Houve erro no título do artigo

p. 2461

onde se lê:

Coesão familiar associada à saúde bucal, fatores socioeconômicos

Family cohesion associated with oral health, socioeconomic factors

leia-se:

Coesão familiar associada à saúde bucal, fatores socioeconômicos e comportamentos em saúde

Family cohesion associated with oral health, socioeconomic factors and health behavior 